American Journal of Pharmaceutical Education 2008; 72 (2) Article 44.

\title{
VIEWPOINTS
}

\section{Diversifying the Team}

\author{
Marie A. Chisholm-Burns, PharmD, MPH \\ The University of Arizona College of Pharmacy
}

How do you define diversity in the departmental or team environment? This question was posed to attendees of a pharmacy practice department heads roundtable session at the American Association of Colleges of Pharmacy (AACP) 2007 Annual Meeting. Most of the responses fit into 1 of 3 categories:

- "Usually based on ethnicity/race. Also considers gender."

- "Valuable differences in members of the team contributed by culture and experiences."

- "Multicultural/racial/gender."

What is most striking about the proffered definitions is that they tend to suggest emphasis on one dimension of diversity: demographics (eg, race, gender). Demographic diversity is, without question, critically important to academia, and much has been written about its significance in the health professions. According to the Institute of Medicine, increased demographic diversity among health professionals is related to greater access to health care in minority patient populations, improved patient options and satisfaction, better communication between health care professionals and patients, and more effective educational experiences for students of health science programs. ${ }^{1}$ Recognizing the significance of demographic diversity in the academic pharmacy setting, AACP set forth an Issue Brief addressing diversity as it relates to pharmacy education, ${ }^{2}$ and the Accreditation Council for Pharmacy Education has incorporated mandates related to diversity in its accreditation standards for academic pharmacy programs including demonstrated commitment (such as a values statement) on the part of the program to encourage faculty and student diversity. ${ }^{3}$

Yet, demographic diversity is only one piece of the larger diversity puzzle in academic pharmacy practice. In fact, this Viewpoint defines diversity in a more encompassing manner, similar to the definition of the Magazine Publishers of America (MPA): "the act of recognizing, appreciating, valuing, and utilizing the unique talents and contributions of all individuals regardless of differences

Corresponding Author: Marie A. Chisholm-Burns, PharmD, MPH, FCCP, FASHP. Professor and Department Head,

University of Arizona College of Pharmacy, 1295 N. Martin Ave., P.O. Box 21202, Tucson, AZ 85721. Tel: 520-626-2298. Fax: 520-626-7355. E-mail: chisholm@pharmacy.arizona.edu or similarities relating to age, color, race, religion, gender, sexual orientation, culture, ethnicity, language, national origin, physical appearance, disability, marital, parental or family status, communication or management style, educational level or background, [and] speed of learning or comprehension." 4 I further expanded the MPA's definition to include experiential diversity, which is integral to academic pharmacy. Experiential diversity in a department of pharmacy practice can be defined as intellectual "depth" developed from a faculty with diverse disciplines/fields and professional and research experiences. Elements of experiential diversity include discipline/field of interest, practice, academic rank/appointment, and work experience. Promoting diversity in academic pharmacy practice is an attempt to reach a critical mass in order to have an intellectually stimulating environment to benefit students, faculty members, and society. Thus, this Viewpoints will address the challenges and benefits of integrating a culture of diversity into academic pharmacy practice departments and set forth recommendations to diversify the team.

There are several challenges to building diversity within academic pharmacy practice. In order to have diversification, we must have a viable pipeline for recruitment. Foremost among the challenges of recruiting faculty members include questionable or lack of interest in academia as a career, lower salary, and increased training requirements. The demand for pharmacy practice faculty candidates is high for several reasons including the $30 \%$ increase in the number of colleges/schools of pharmacy in the past 20 years, as well as increased class enrollment among established colleges/schools of pharmacy. ${ }^{5}$ Further restricting the pool of potential candidates is competition from the private sector, which offers salaries generally well above those found in academia (with sign-on and other bonuses frequently available). The salary disparities often becomes a greater point of contention when considering that the private sector hires many individuals directly out of professional degree programs, whereas many positions in academia require postgraduate training (eg, residency, specialty residency, fellowship) in addition to professional and/or graduate degrees. Recruitment as it relates to demographic diversity is even more difficult due to the small and decreasing number of minorities in the pipeline. According to AACP's 2006-2007 Profile 


\section{American Journal of Pharmaceutical Education 2008; 72 (2) Article 44.}

of Pharmacy Faculty, ${ }^{6}$ minority group members represent only $25 \%$ of pharmacy faculty members, and the hopes of many concerning increased numbers of minority faculty members in the near future are cast with doubt and languish due to the limited pool of potential candidates. For example, in the 2005-2006 academic year, underrepresented minorities received only $12 \%$ of the PharmDs conferred and less than $6 \%$ of the PhDs conferred. ${ }^{7}$ Despite the barriers to demographic diversity currently faced by academic pharmacy, its value cannot be overstated as demographic diversity enhances health science academia and its impact on external health care systems by promoting: (1) cultural competence among faculty members and students which will, in turn, facilitate the provision of culturally competent health care to patients; (2) research opportunities that address the needs of minority and underrepresented populations; and (3) increased representation of minority group members and women among health care practitioners. ${ }^{8}$

Related to the issue of recruitment is the difficulty in achieving a balance in faculty ranking, defined as having adequate numbers of junior and senior faculty members. This struggle for balance is particularly relevant to newer or growing academic pharmacy practice departments. An examination of the faculty rosters of 5 schools/colleges of pharmacy that opened within the last 5 years found that among pharmacy practice faculty members, junior faculty members generally outnumbered senior faculty 6 to 1. The balance of having adequate numbers of senior and junior faculty members is important for several reasons. For example, superior senior faculty members may provide positive mentoring experiences to junior faculty by guiding them to work more effectively, helping them to achieve promotion and tenure, sharing teaching and classroom problem solving techniques, providing research/ scholarship strategies (eg, project planning, publication, grantsmanship), and sharing strategies to achieve life balance in academia. Ideally, there should be at least 1 senior faculty member available to provide mentoring to each junior faculty member in the department. For those of us who have interacted and mentored junior faculty members, we can attest that many of them bring a new energy to the department and this energy often helps to reinvigorate senior faculty mentors. Junior faculty members are and should be viewed as the future of academic pharmacy, and appropriate resources should be invested in their development. Implementing mentoring programs and providing resources for development demonstrates a department's commitment to this important endeavor. Careful attention should be given to the chasm in the ratio of junior to senior faculty members so as not to erode the benefits of senior members serving as mentors to junior faculty members.
In addition to mentoring, there are other benefits to having experiential diversity within an academic pharmacy department. For example, experiential diversity is a crucial tool in promoting the quality of faculty life within the academic pharmacy department. Research indicates that pharmacy faculty members believe that it is important to work in an intellectually challenging environment, ${ }^{9}$ and a department composed of faculty members with diverse interests and experiences is more likely to foster a dynamic, intellectually stimulating workplace. To explain further, diversity of disciplines/fields of interest and related practice/work experience within a department enhances potential collaborative partnerships between faculty members, as faculty members are more likely to identify colleagues whose knowledge, skills, and interests are complementary, but not necessarily repetitive. For example, one faculty member with a strong clinical background may collaborate with a faculty member who specializes in economics on a project that examines the therapeutic and economic outcomes of interventions. Further, having diverse faculty members in a department increases the potential for developing collaborations with organizations such as centers of excellence that are within the University but outside the department and/or college/school. These external collaborations may increase the department's access to resources (such as equipment housed in core research facilities) as well as increase faculty success and job satisfaction.

Fostering an experientially diverse faculty also increases opportunities to amass internal resources to benefit the department such as greater indirect costs and funds generated through clinical service contracts. These resources can be used to support departmental functions that are otherwise lacking in means, as well as to support the teaching mission of the department and college. Similarly, a department comprised of faculty members with diverse experiential backgrounds may help redress myopic vision and planning, and provide solutions to problems that have been viewed as unsolvable. In other words, experiential diversity opens the door to multiple paths to achieving the department's mission and vision. However, the introduction of too many viewpoints into the department's decision-making process regarding resource allocation, mission, vision, strategic planning, and day-to-day operations may result in some discordance and a decreased ability to quickly respond - in other words, the overload of input may slow departmental decisions. To minimize these conflicts while encouraging team building, and to find ways to discuss differences without dividing the departmental community, it is important to respect each faculty member's viewpoint, position, and department role, and to have strategies in place to allow faculty members to negotiate disagreements and reach resolutions. 


\section{American Journal of Pharmaceutical Education 2008; 72 (2) Article 44.}

For example, strategies may include: (1) providing structured opportunities for respectful dialogue regarding possible resolutions; (2) setting a reasonable schedule for decision-making to ensure that resolutions are produced in a timely manner; and (3) allowing opportunities for all faculty members to participate in the decision-making process. By applying these strategies, diverse views may productively contribute to departmental operations and logistics, with solutions that better serve and represent the entire department.

To further optimize advantages and overcome challenges to developing a culture of diversity in academic pharmacy practice, the following strategies are recommended to department administrators:

(1) Integrate diversity into the mission and vision of the department. ${ }^{10}$

(2) Help define and demonstrate how each department faculty member fits into the overall mission and vision of the department. This philosophy should also guide new hires and the faculty search process.

(3) Practice inclusion and building collegiality among faculty members. ${ }^{11}$

(4) Facilitate effective mentoring relationships between faculty members. ${ }^{11}$

(5) Encourage and facilitate cross-discipline or -field collaborations between faculty members.

(6) Utilize the diverse knowledge and skills of faculty members to help identify resource allocation needs and priority areas within the department.

(7) Review, identify, and correct disparities and bias in salary/resource distribution, performance evaluation, and promotion and tenure opportunities. ${ }^{11}$

Perhaps the most essential step in promoting diversity is building a sense of community and shared vision within the department. A top-down approach (mandates and policies issued by the administration to enact change) will only go so far in building department diversity and may be met with intense opposition that will hinder diversity efforts. Strategies implemented to improve diversity cannot affect a long-term shift in departmental culture if they are not embraced and supported by faculty members. Thus, for diversity efforts to have a lasting impact on the department, multiple approaches must be taken that include both the administration and the faculty. In other words, faculty members must take shared responsibility for diversity efforts, and actively participate in and facilitate the promotion of diversity throughout all departments. Faculty members and the administration must work together as a team, as a community, to build and sustain the diversity of their department.

In conclusion, diversity is essential to the academic pharmacy practice department as it: (1) creates and sustains a culturally competent pharmacy workforce; (2) advances research regarding the health and well-being of underrepresented groups; (3) enhances the learning and research environment; and (4) enriches the quality of faculty life and the department workplace. There are challenges to improving diversity in the academic department setting; however, they are not insurmountable. A few recommendations to address these challenges and develop diversity were offered in this viewpoint. Faculty and administration should pursue an ongoing dialogue which includes issues such as how diversity is defined in the department environment, goals of diversity, and the possible impact of diversity on the department at macro and micro levels, pharmacy as a profession, and the populations we serve. Additional planning and resources to bolster the presence of demographic and experiential diversity in the academic setting should also be identified and utilized. Thus, by working together, the administration and the faculty can advocate and implement fundamental changes that support and preserve a culture of diversity within pharmacy practice.

\section{REFERENCES}

1. Institute of Medicine. In the nation's compelling interest: ensuring diversity in the healthcare workforce. Washington, DC: Academic Medicine; 2004.

2. American Association of Colleges of Pharmacy. Issue brief: diversity in health professions. 2005. Available at http:// www.aacp.org/site/tertiary.asp?TRACKID $=\& \mathrm{VID}=2 \& \mathrm{CID}=880$ $\& D I D=6692$. Accessed February 4, 2008.

3. Accreditation Council of Pharmacy Education (ACPE).

Accreditation standards and guidelines for the professional program in pharmacy leading to the Doctor of Pharmacy degree. Chicago, IL: ACPE; 2006. Available at http://www.acpe-accredit.org/pdf/

ACPE_Revised_PharmD_Standards_Adopted_Jan152006.pdf. Accessed February 4, 2008.

4. Magazine Publishers of America. Focusing on diversity. Available at http://www.magazine.org/content/files/focusingondiversity.pdf. Accessed February 4, 2008.

5. Student Applications, Enrollment \& Degrees Conferred. Alexandria, VA: American Association of Colleges of Pharmacy: 2007. Available at: http://www.aacp.org/site/page.asp?TRACKID= $\& \mathrm{VID}=1 \& \mathrm{CID}=106 \& \mathrm{DID}=3113$ Accessed on April 14, 2008. 6. American Association of Colleges of Pharmacy. 2006-07 Profile of Pharmacy Faculty. AACP Institutional Research Report Series; 2006. 7. American Association of Colleges of Pharmacy. Profile of Pharmacy Students. AACP Institutional Research Report Series; 2007. 8. King TE Jr., Dickinson TA, DuBose TD Jr., et al. The case for diversity in academic internal medicine. Am J Med 2004;116:284-9. 9. Spivey CA, Chisholm-Burns MA, Murphy JE, Rice L, Morelli C. Assessment of and recommendations to improve faculty satisfaction and retention. Unpublished manuscript.

10. Stanley JM, Flynn Capers C, Berlin LE. Changing the face of nursing faculty: minority faculty recruitment and retention. J Prof Nurs. 2007;23:253-61.

11. Wesson DE, King TE Jr., Todd RF, et al. Achieving diversity in academic internal medicine: recommendations for leaders. Am J Med. 2006;119:76-81. 